\title{
Digestive Decolonization of Colorectal Carriage of Vancomycin-resistant Enterococcus faecium in a Japanese Adult: A Case Report
}

\author{
Shunsuke Uno ${ }^{1,2}$, Yaoko Takano ${ }^{2}$, Osamu Iketani ${ }^{2}$, Tomohiro Abiko ${ }^{3}$, Tomoru Miwa ${ }^{3}$, \\ Kosaku Nanki ${ }^{4}$, Tomohiro Kurihara ${ }^{2,5}$, Yuko Tamura ${ }^{2}$, Miyuki Ara $^{2}$, Yoshifumi Uwamino ${ }^{2,6}$, \\ Masayoshi Shinjoh ${ }^{2,7}$, Takehiko Mori ${ }^{2,8}$ and Naoki Hasegawa ${ }^{1,2}$
}

\begin{abstract}
:
Patients with vancomycin-resistant Enterococcus (VRE) colonization should be managed in an isolation room with contact precautions. We herein report a patient whose colorectal carriage of VRE was successfully decolonized using concomitant bowel irrigation with polyethylene glycol, probiotics, and oral antimicrobials, linezolid and orally-administered daptomycin, for release from isolation and contact precautions. We therefore would like to suggest a potential strategy for managing patients with VRE colonization.
\end{abstract}

Key words: vancomycin-resistant Enterococcus, decolonization, isolation precautions

(Intern Med Advance Publication)

(DOI: 10.2169/internalmedicine.6088-20)

\section{Introduction}

The prevalence of vancomycin-resistant Enterococcus (VRE) is increasing worldwide $(1,2)$, and VRE infections pose a severe threat to public health. VRE bacteremia is associated with a higher case fatality and longer hospital stay than vancomycin-susceptible Enterococcus species (3). Patients with VRE colonization are managed by implementing isolation precautions in medical facilities (4). However, rehabilitation hospitals and long-term care facilities with expertise in hygiene practices and sufficient paramedical staff that adhere to these isolation protocols for managing multidrugresistant organisms (MDROs), including VRE, are limited in Japan.

Managing patients who need to be hospitalized following the detection of an MDRO in a culture specimen is a serious infection control concern, even though spontaneous decolonization is noted in $48.2 \%$ of MDRO colonization cases (5). VRE decolonization is a potential choice for preventing hospital transmission of VRE. However, efficient decolonization strategies have not yet been established, although the potential efficacy of fecal microbiota transplantation (FMT) $(6,7)$ and antimicrobial decolonization regimes (8) have previously been reported.

We herein report a patient treated using concomitant bowel irrigation with polyethylene glycol (PEG), probiotics, and the oral administration of the antimicrobials, linezolid and daptomycin, for VRE decolonization.

\section{Case Report}

A 60-year-old man with no relevant medical history was admitted to Keio University Hospital, a tertiary-care hospital

\footnotetext{
${ }^{1}$ Department of Infectious Diseases, Keio University School of Medicine, Japan, ${ }^{2}$ Division of Infectious Diseases and Infection Control, Keio University Hospital, Japan, ${ }^{3}$ Department of Neurosurgery, Keio University School of Medicine, Japan, ${ }^{4}$ Division of gastroenterology, Department of Internal Medicine, Keio University School of Medicine, Japan, ${ }^{5}$ Department of Emergency and Critical Care Medicine, Keio University School of Medicine, Japan, ${ }^{6}$ Department of Laboratory Medicine, Keio University School of Medicine, Japan, ${ }^{7}$ Department of Pediatrics, Keio University School of Medicine, Japan and ${ }^{8}$ Division of hematology, Department of Internal Medicine, Keio University School of Medicine, Japan
}

Received: August 11, 2020; Accepted: April 12, 2021; Advance Publication by J-STAGE: June 26, 2021

Correspondence to Dr. Naoki Hasegawa, n-hasegawa@z8.keio.jp 


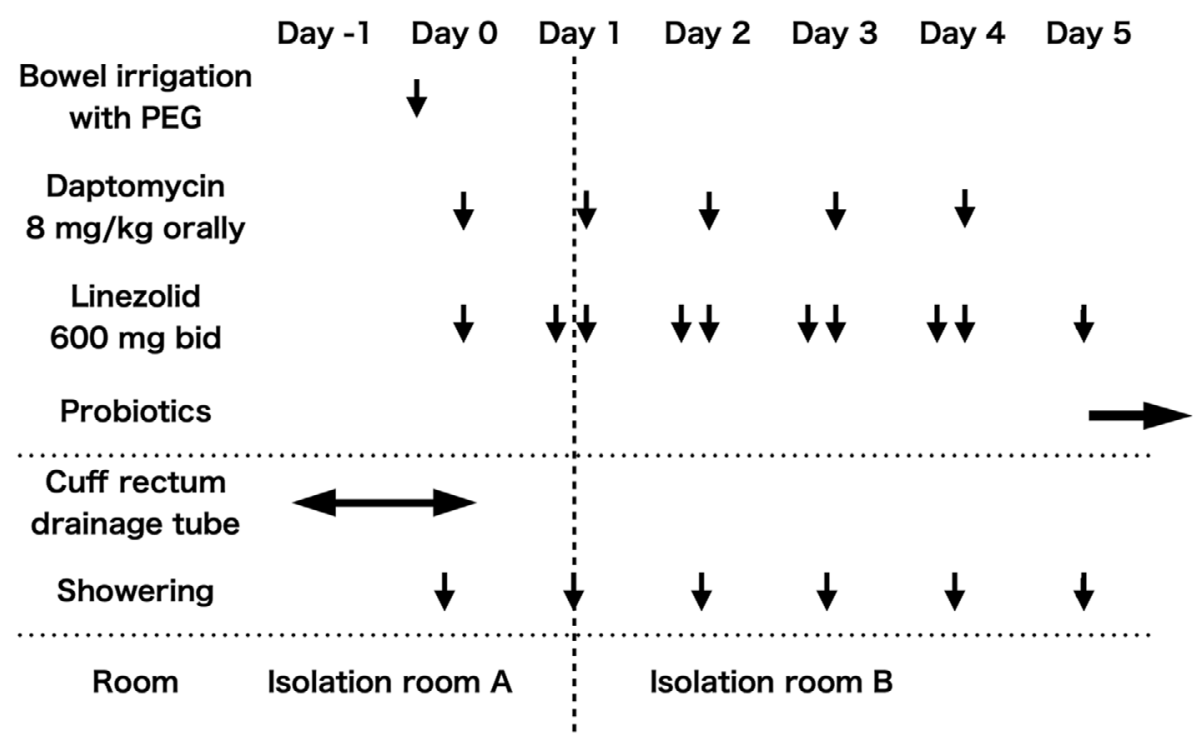

Figure. Protocol for vancomycin-resistant Enterococcus faecium decolonization. PEG: polyethylene glycol

with 1,044 beds, for acute aortic dissection involving right brain infarction. Despite emergency ascending aortic arch replacement and extracranial decompression on the day of admission, he suffered from left hemiparesis and altered consciousness, after which he was bed-ridden and required nursing care. Two months later, surgical site infection was observed following skull reconstruction (using thigh fascia lata), for which the patient was prescribed broad-spectrum antimicrobial therapy for eight weeks.

The patient was transferred to the rehabilitation hospital a further two months later. However, he was immediately readmitted to our hospital because of vancomycin-resistant Enterococcus faecium colonization, as confirmed by a rectal swab culture. Polymerase chain reaction was performed using Xpert vanA/vanB (Cepheid, Sunnyvale, CA, USA) for the recovered VRE colony, and the presence of the vanA gene was confirmed. The minimum inhibitory concentration of daptomycin and linezolid for this strain was $2 \mathrm{mg} / \mathrm{dL}$ and $<2 \mathrm{mg} / \mathrm{dL}$, respectively. We implemented contact isolation precautions and checked rectal swab cultures for the clearance of VRE colonization.

The patient needed further rehabilitation to improve his activities of daily living. However, no rehabilitation hospital or long-term care facilities would admit him owing to their lack of expertise in the necessary hygiene practices and insufficient paramedical staff able to adhere to the isolation precautions. VRE was detected in rectal swab cultures for one year following re-admission to our hospital. Owing to the prolonged hospital stay and unsuccessful spontaneous clearance of VRE, VRE decolonization was considered.

A decolonization protocol was prepared (Figure) by referring to a previous report (8). The protocol was approved by the clinical ethics consultation of Keio University Hospital (2018-13). The patient was isolated in room A before decolonization. A naso-duodenal (ND) tube was inserted three days before decolonization, and a cuff rectum drainage tube was inserted a day before decolonization was performed by bowel irrigation with PEG. Two liters of PEG was infused via the ND tube over six hours to wash out the bowel contents, and we noted that the defecated bowel content was a clear fluid on the starting day (Day 0). The patient was bathed with soap and shampoo, and his clothes and linens were replaced every day. A five-day dose of antimicrobials against VRE was prescribed that included linezolid $600 \mathrm{mg}$ twice a day and orally administered daptomycin $8 \mathrm{mg} / \mathrm{kg} /$ day. Enteral feeding was stopped on Day 0 and restarted on Day 1.

The patient was transferred to isolation room B after bathing on Day 1. Removal of the cuff rectum drainage tube was planned on the evening of Day 0; however, the removal was deferred to Day 7 due to prolonged diarrhea. Probiotics administration, involving a mixture of the products derived from E. faecalis and Bacillus subtilis, Biofermin powder (Biofermin Pharm, Hyogo, Japan) 9 g/day, divided into 3 doses; and Clostridium butyricum, Miya-BM fine granules (Miyarisan Pharm, Tokyo, Japan) $3 \mathrm{~g} /$ day, divided into 3 doses, was initiated after the completion of antimicrobial therapy. Probiotics were continued until the patient was discharged.

Rectal swab cultures were obtained at planned intervals starting at one week, one month, three months, and six months after initiating the decolonization protocol on VRE Selective Agar (Becton Dickinson, Franklin Lakes, NJ, USA). VRE was not detected in any of the rectal swab cultures despite two-week antimicrobial treatment with cefepime following piperacillin/tazobactam for a urinary tract infection four months after decolonization. No severe adverse events were observed during the decolonization process; however, the removal of the cuff rectum drainage tube was deferred until Day 7 because of prolonged diarrhea. The 
introduction of this protocol allowed a cost-savings of $1,690,732$ yen (approximately 15,650 USD) per year in gowns and gloves, which was calculated by multiplying the actual price delivered to the hospital by the estimated number of gloves and gowns used in 1 year, while 281,000 yen (approximately 2,600 USD) was spent on instruments and drugs. The number of gloves and gowns used per year was estimated by multiplying the average number of gloves and gowns used per week by 52 .

\section{Discussion}

We reported our management of a patient who underwent decolonization of VRE using an antimicrobial method after an extended period of compliance with contact isolation precautions and unsuccessful spontaneous VRE clearance. VRE was not detected in the rectal swab cultures for six months after decolonization, and no severe adverse events were observed, indicating successful decolonization. Thus, the isolation measures were relaxed. We also saved approximately 1.41 million yen (approximately 13,053 USD) per year by reducing the use of personal protective equipment for contact precautions after deducting the price of the instruments and drugs used for decolonization.

Managing patients with VRE colonization is a serious challenge in infection control. Some studies have suggested that patient isolation may not be the best measure of preventing infection $(9,10)$. However, these reports are based on the endemicity of VRE on different levels. Data from the Japan Nosocomial Infections Surveillance suggest that the annual recovery rate of VRE from 2013 to 2018 was < $1 \%(11,12)$. Even a single detection at a facility, irrespective of whether it causes infectious disease, should be considered an outbreak. Based on these data, the discontinuation of isolation precautions in Japan should be duly considered. Owing to the limited number of facilities with expertise in hygiene and sufficient paramedical staff that strictly follow isolation precautions in Japan, decolonization is a meaningful strategy for achieving infection control.

Decolonization of VRE using antimicrobials is challenging. Selective bowel decolonization is reportedly effective in only $35 \%$ of liver or kidney transplant patients (13). One study mentioned an improved method that combined antimicrobials against Enterococcus spp. with mechanical preparations of PEG, which is commonly used before colonoscopy (8). To reduce the total bacterial burden and increase the probability of successful decolonization, our protocol was devised by referring to the methods used in a previous study (8).

Another study reported not only re-colonization of VRE after hematopoietic stem cell transplantation but also showed that exposure to antimicrobials may increase the likelihood of re-colonization (14). Our patient's rectal swab cultures remained negative despite the administration of piperacillin/tazobactam and cefepime for urinary tract infection four months after decolonization. This implies that the regimen followed in this case markedly decreased the intestinal bacterial load of VRE. As mentioned in several studies, clearance of VRE colonization is controversial, and it may take up to three or four consecutive negative cultures one or more weeks apart to identify successful decolonization $(5,7,13,14)$. Thus, to confirm successful decolonization, we obtained rectal swab cultures until six months after decolonization, as previously reported (6), using a selective medium with increased sensitivity of detection.

The criteria of which patients should be decolonized and the strategy of decolonization have not yet been established. Although there are no reports that clearly indicate how long an observation period is required to achieve spontaneous clearance, a previous systematic review showed that spontaneous clearance of VRE colonization was seen in half of cases at 26 weeks from the time of documented colonization (15). Another retrospective study showed that $56.8 \%$ of patients with VRE colonization demonstrated clearance within a median follow-up duration of 382 days (16). Therefore, if spontaneous clearance is not obtained within 6 to 12 months, it might be reasonable to consider decolonization, depending on the need. A previous report suggested two strategies-FMT and an antimicrobial method-for decolonizing VRE (6-8); the advantages of the antimicrobial decolonization method may be its simplicity and acceptance by the patient and family. We presented both options to this patient, and the family chose the antimicrobial method. The disadvantage of antimicrobial decolonization is the possibility of side effects. Daptomycin is not likely to be a problem because of its low oral bioavailability (17); however, linezolid may cause high rates of thrombocytopenia in Japanese patients after use for more than a week (18). In addition, further resistant bacteria might emerge due to the use of antimicrobials. However, in the present case, the risk of either of these occurring was not considered high, as both were administered for only five days.

In conclusion, this report suggests a potential strategy for decolonizing colorectal VRE to manage patients with VRE colonization in resource-limited medical facilities.

\section{The authors state that they have no Conflict of Interest (COI).}

\section{Funding source: None}

Ethics approval: The institutional review board of Keio University School of Medicine approved this study (20170385), and informed consent was obtained from the next of kin of the patient.

\section{Acknowledgement}

We thank the medical technicians of the microbiological laboratory of Keio University Hospital for performing the colorectal swab cultures and gene test.

Author contributions: Shunsuke Uno designed the study, analyzed the data, and drafted the manuscript; Yaoko Takano de- 
signed the study and analyzed the data; Osamu Iketani designed the study, applied for ethics approval from the institutional review board, and performed clinical ethics consultation; Tomohiro Abiko and Tomoru Miwa were doctors in charge of the patient; Kosaku Nanki and Tomohiro Kurihara were consultants for patient management; Yuko Tamura, Miyuki Ara, Yoshifumi Uwamino, Masayoshi Shinjoh, and Takehiko Mori confirmed the analyses and helped edit the manuscript; Naoki Hasegawa conceptualized the study, revised the article for intellectual content, and acquired funding. All authors read and critically revised the first as well as the subsequent and final drafts of this manuscript.

\section{References}

1. Vehreschild M, Haverkamp M, Biehl LM, Lemmen S, Fatkenheuer G. Vancomycin-resistant enterococci (VRE): a reason to isolate? Infection 47: 7-11, 2019.

2. Remschmidt C, Schroder C, Behnke M, Gastmeier P, Geffers C, Kramer TS. Continuous increase of vancomycin resistance in enterococci causing nosocomial infections in Germany - 10 years of surveillance. Antimicrob Resist Infect Control 7: 54, 2018.

3. Prematunge C, MacDougall C, Johnstone J, et al. VRE and VSE Bacteremia Outcomes in the Era of Effective VRE Therapy: A Systematic Review and Meta-analysis. Infect Control Hosp Epidemiol 37: 26-35, 2016.

4. Siegel JD, Rhinehart E, Jackson M, Chiarello L; Healthcare Infection Control Practices Advisory Committee. Management of multidrug-resistant organisms in health care settings, 2006. Am J Infect Control 35 (10 Suppl 2): S165-S193, 2007.

5. Davido B, Moussiegt A, Dinh A, et al. Germs of thrones - spontaneous decolonization of Carbapenem-Resistant Enterobacteriaceae (CRE) and Vancomycin-Resistant Enterococci (VRE) in Western Europe: is this myth or reality? Antimicrob Resist Infect Control 7: 100, 2018.

6. Bilinski J, Grzesiowski P, Sorensen N, et al. Fecal Microbiota Transplantation in Patients With Blood Disorders Inhibits Gut Colonization With Antibiotic-Resistant Bacteria: Results of a Prospective, Single-Center Study. Clin Infect Dis 65: 364-370, 2017.

7. Dinh A, Fessi H, Duran C, et al. Clearance of carbapenemresistant Enterobacteriaceae vs vancomycin-resistant enterococci carriage after faecal microbiota transplant: a prospective comparative study. J Hosp Infect 99: 481-486, 2018.
8. Cheng VC, Chen JH, Tai JW, et al. Decolonization of gastrointestinal carriage of vancomycin-resistant Enterococcus faecium: case series and review of literature. BMC Infect Dis 14: 514, 2014.

9. Bearman G, Abbas S, Masroor N, et al. Impact of Discontinuing Contact Precautions for Methicillin-Resistant Staphylococcus aureus and Vancomycin-Resistant Enterococcus: An Interrupted Time Series Analysis. Infect Control Hosp Epidemiol 39: 676-682, 2018.

10. Martin EM, Bryant B, Grogan TR, et al. Noninfectious Hospital Adverse Events Decline After Elimination of Contact Precautions for MRSA and VRE. Infect Control Hosp Epidemiol 39: 788-796, 2018.

11. Tsutsui A, Suzuki S. Japan nosocomial infections surveillance (JANIS): a model of sustainable national antimicrobial resistance surveillance based on hospital diagnostic microbiology laboratories. BMC Health Serv Res 18: 799, 2018.

12. Ministry of Health Labour and Welfare. JANIS (Japan Nosocomial Infections Surveillance) [Internet]. Available from: https://janis.mhl w.go.jp/english/index.asp.

13. Patel R, Allen SL, Manahan JM, et al. Natural history of vancomycin-resistant enterococcal colonization in liver and kidney transplant recipients. Liver Transpl 7: 27-31, 2001.

14. Hughes HY, Odom RT, Michelin AV, et al. A retrospective cohort study of antibiotic exposure and vancomycin-resistant Enterococcus recolonization. Infect Control Hosp Epidemiol 40: 414-419, 2019.

15. Shenoy ES, Paras ML, Noubary F, Walensky RP, Hooper DC. Natural history of colonization with methicillin-resistant Staphylococcus aureus (MRSA) and vancomycin-resistant Enterococcus (VRE): a systematic review. BMC Infect Dis 14: 177, 2014.

16. Farfour E, Si Larbi AG, Couturier J, et al. Asymptomatic carriage of extensively drug-resistant bacteria (eXDR), a simple way to assess spontaneous clearance. J Hosp Infect 104: 503-507, 2020.

17. Estes KS, Derendorf H. Comparison of the pharmacokinetic properties of vancomycin, linezolid, tigecyclin, and daptomycin. Eur J Med Res 15: 533-543, 2010.

18. Takahashi Y, Takesue Y, Nakajima K, et al. Risk factors associated with the development of thrombocytopenia in patients who received linezolid therapy. J Infect Chemother 17: 382-387, 2011.

The Internal Medicine is an Open Access journal distributed under the Creative Commons Attribution-NonCommercial-NoDerivatives 4.0 International License. To view the details of this license, please visit (https://creativecommons.org/licenses/ by-nc-nd/4.0/).

(C) The Japanese Society of Internal Medicine Intern Med Advance Publication 